\title{
Interactive comment on "Using StorAge Selection functions to quantify ecohydrological controls on the time-variant age of evapotranspiration, soil water, and recharge" by Aaron A. Smith et al.
}

\section{Aaron A. Smith et al.}

aaron.smith@abdn.ac.uk

Received and published: 4 June 2018

\section{Reviewer 2 General Comments}

In this article, the authors would like to propose a new method to quantify ecohydrological controls based on time-variant water ages. The main novelty of this study is using the mass balance of isotopes from different water sources (rainfall, xylem and soil water) to characterise retention times for different storages. The research questions of the work are interesting. However, (1) the proposed methods are poorly explained, (2) the model assumptions and results are not properly tested and evaluated, and (3) the overall writing can be better. I would like that the authors can evaluate their method by

Printer-friendly version

Discussion paper 
a comprehensive sensitivity study, so that I suggest a major revision.

\section{Response to Reviewer 2 General Comments}

The authors thank Reviewer 2 for their comments. As discussed more in the response to specific comments, the authors will revise the methods section to improve clarity of how the equations were applied and used to solve the water, mass, and age balance equations. Additional justification for the model structure will be incorporated into the methods section to aid in the evaluation. As suggested by the reviewer, a parameter sensitivity analysis will be included to show how well the model performs.

\section{Reviewer 2 Specific Comments}

R2C1: A label is used for multiple variables. For an example, all the " $T$ "s are confusing; P4 Ln 14 the absolute age (T); P5 Ln 25 the isotopic composition of water ranked by age (T); P5 Ln 29 evaporation fractionation for each water age $(T)$

Response to $\mathbf{R 2 C 1}$ : The original definition provided ( $T$ is the absolute water age) is the appropriate definition and the use of $T$ in the other statements was intended to distinguish the general term "age" for absolute vs relative. In our study we define the absolute age as the elapsed time water has spent in the modelling domain, while the relative time is the elapsed time water has spent in a control volume (a given soil layer). As this is unclear, the general term "age" will be accompanied by "absolute" or "relative" where appropriate.

R2C2: Different labels are used for a variable. For an example, when Precipitation is defined to be "J" in P3 Ln 9, Figure 1 uses P.

Response to R2C2: Figure 1 will be updated for consistency with the label names (eg. $P$ vs J). Since $P$ is later defined as a probability, its use in the figure was a typo.

Printer-friendly version

Discussion paper

R2C3: The word choice can be strange. In P3 Ln15 and Ln18, "Diffusion (D)" is an odd choice. Can it be "infiltration", "recharge" or "surface flow"? Honestly, I don't know. Maybe, it will be clearer if the authors can label it on Figure 1. I feel that "control 
volume" or "CV" is a jargon. Can the author just say a "compartment", "gird", "cell" or "element" instead?

Response to R2C3: Figure 1 will also be updated to explicitly show the fluxes within each control volume. This visual aid should help to resolve confusion on the terminology used in the water balance (ie. Diffusion). As suggested by the reviewer, the term "control volume" will be changed to soil layer to be more unambiguous, and more representative of the modelled soil water volume.

R2C4: There are some unnecessary abbreviations. For an instance, "kernel density estimation" (KDE) only appeared two times in the article. When I read KDE in P14 Ln 18, I needed to try to see what KDE is in P9 Ln10.

Response to R2C4: A table of abbreviations with symbols will be included in the supplementary material. This will aid in the readability of the manuscript given the large number of symbols used for fluxes, water ages, and isotopic compositions. The use of KDE as an abbreviation will be removed.

R2C5: The definitions are bad. I don't understand what "T (CV from 0 to delta) or zeta (CV below delta z)" are in P5 Ln 4, because I don't understand why absolute age (T), relative age (zeta), depth (delta z) and Control volume (CV) are related. I don't know how high soil and low soil moistures are defined in Figure 6 . All of these make the theory and methodology session difficult to read. The whole article should be revised with a better notation housekeeping.

Response to R2C5: The relationship between the absolute and relative age distributions was previously provided in the manuscript (Eq. 3 and P4 Lns 13-16). However, as noted in Response to R1C8, the authors recognize that the units provided in the equation and the definition of $\omega_{J}$ may lead to confusion. The definition (and relationship between the absolute and relative age) will be modified for clarity.

R2C6: In P17 Ln 22: uncertainty is recognised to be important, but nothing has been

Printer-friendly version

Discussion paper 
done in terms of model structures and model parameters. The data quality issues were not explored. At the moment, the results are based on the face values of outputs from a complicated model based on some coarse-temporal data. At a result, the assumptions of the models are poorly validated. In fact, the isotope model results are very bad in Figure 3b-i. How these poor results affect the overall retention results should be quantified by a sensitive study.

Interactive

Response to R2C6: The authors respectfully disagree that the model results are poor. Also, whilst monthly soil water and xylem is "coarse" it is still an unusually rich data set over a year-long period to test the model with. The calibration of 4 soil layers using one downward flow SAS function (3 parameters) to achieve NSE values $>0.4$ for $\delta^{2} H, \delta^{18} \mathrm{O}$, and Ic-excess shows reasonable functionality of the model to capture feedforward flow processes. The authors recognize that the abstract nature of probability functions within the model does not aid in interpretation of the parameter sensitivity without a more formal analysis. For this reason, the authors will include a parameter sensitivity analysis in the appendix of the revised manuscript.

R2C7: The author recognised the data issues in P20 Ln14. However, they did not do much about it. The uncertainty related to data measurements is not quantified. How measurement errors affect the retention results was not studied. The justification for using Ic-excess is weak (P8 Lns 27-28)

Response to R2C7: Again, we respectfully disagree with the reviewers comment; the authors directly included the measurement uncertainty in the analysis of the model performance (Eq. 19). The model has high weighting to simulate the temporal periods with low measured variability, and lower weighting when measurement variability is high. In order to directly assess how the measurement error would influence the retention results, recalibration would be required with a different efficiency criteria that does not include the measurement uncertainty. Other evaluation tools to include measurement uncertainty (e.g. Kuppel et al., 2013), use a similar weighting method (e.g. covariance matrices) to reduce the influence of high uncertainty days on the overall model

Printer-friendly version

Discussion paper 
performance. As this is essentially a sensitivity analysis of how well efficiency criteria perform with input measurements, the authors believe that this analysis would not significantly contribute to the manuscript. Lc-excess was used within this study to ensure that evaporative fractionation was simulated appropriately. Using only $\delta^{2} \mathrm{H}$ and $\delta^{18} \mathrm{O}$ as calibration metrics may result in slight bias of each isotope and result in incorrect evaporation estimation. For example, a slight bias for more enriched $\delta^{2} H$ and slight bias for more depleted $\delta^{18} O$ may provide reasonable efficiency; however, would result in a large difference in the Ic-excess from measured values. This additional justification will be added to the manuscript.

R2C8: At the moment, the authors are subjectively selected its model structure without much evaluation. They need to provide results to show how different variables (e.g. different soil water depths) should be included in the proposed method. The authors should explain how different model structures affect the retention results.

Response to R2C8: As discussed in the initial Response to Reviewer 2, the model structure was carefully constructed to mirror data availability. The simulated depths were chosen based on the measured data (at $5 \mathrm{~cm}$ intervals). Smaller layers would result in more numerical instability and would require smaller time-step to avoid the instabilities (< 1 day). Using larger soil storage would likely result in lost measurement information. For example, at $5 \mathrm{~cm}$ there is greater evidence of evaporative enrichment than is observed at $10 \mathrm{~cm}$. The integration of these two layers would result in the loss of information of greater preference for evaporation to occur from the upper $5 \mathrm{~cm}$ (Fig $5 c$ and $5 d)$.

R2C9: Because using isotopes is the main novelty here, I want to see more evaluation for the isotope model selection. I want to see how it was set up. I want to know how different models replacing Equations 8 or 9 can affect the overall retention age results. I also want to see how the sensitivity of the model parameters of Equations 8 or 9 affects the storage retention. 
Response to R2C9: The Craig-Gordon model is the standard equation used for isotopic fractionation, which was developed through the use of empirical equations. These equations are all explained through physical principals and changing the use of these equations for this study would require significant experimental work well beyond the scope of the modification of SAS functions presented here. As shown in the initial Response to Reviewer 2, the parameters used within the Craig-Gordon model may have some sensitivity within the model results. These parameters will be included in the sensitivity analysis (sensitivity analysis described in Response to R2C6).

R2C10: Similarly, different distributions should be tests for the hydrology part. For example, in addition to the beta distribution in P7 Ln 24, the author should try gamma and other distributions. In fact, the authors have some ideas about it in P19 Ln 10. The authors should explore the sensitivity of parameters (both constant and time-variance) in Equation 14 (P7 Lns 26-27). The temporal scale of data is very coarse.

Response to R2C10: The selection of the beta distribution as the most representative model structure was based on the flexibility and functionality of the beta distribution to reproduce shapes similar to a linear distribution, uniform distribution, exponential distribution, and gamma distribution while simultaneously producing distribution shapes unique to the beta distribution. The uniqueness of the beta distribution is shown in the initial Response to Reviewer 2.

R2C11: The model includes processes across different temporal scales. This kind stiff model has a lot of numerical stability issues because of different scales. The authors recognised some stability problems in P9 Ln 19. Explaining how different processes scales affects the stability of the numerical schemes can be useful information for possible model users to know the technical issues related to the implemented model. The authors should illustrate how their numerical schemes may introduce artefacts. The authors need to try different numerical scheme to illustrate that their parameter estimates are not biased. Overall, the above model issues can be resolved by a comprehensive sensitivity study. Of course, the authors can also do some laboratory or field experi-

Printer-friendly version

Discussion paper

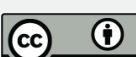


ment to validate model assumptions at Heather Site A and B.

Response to R2C11: The authors are unclear on what the reviewer means by "stiff model" or is intending when suggesting the model includes processes at different temporal scales. The model was run on daily time-steps and uses daily data for the simulations. Numerical instabilities would only occur during the high flow conditions if the time-step is not adjusted. These high flow conditions occurred very rarely, only once throughout the simulation during a 1-in-200 year precipitation event. During these periods, the fluxes were estimated using the daily time-step for consistency with the other time-steps, and discretized to the time-step required to meet the Courant criteria. During model development, sensitivity analysis of the SAS functions with various timesteps was tested to ensure that there would not be a significant deviation to larger time-steps. At sites where the change in soil moisture is greater, significant consideration of the long-term time-step is require to ensure that the large changes in volume over a small time-step are captured. The authors will briefly discuss this consideration in the discussion section (Section 4.3).

R2C12: The discussion is a bit haphazard. For an instance, I don't understand why the fractionation of xylem water is discussed in P18 Lns 12-18. I am not sure whether the proposed model can address this fractionation issue, or it is a limitation of the proposed model. When the authors discuss ecohydrological and hydrological controls of their sites, they need to link them to their test methods and their results. Currently, Sections 4.1 and 4.2 are like a literature review. The conclusion is very weak. The authors just said "improved understanding" in P20 Ln 29. After the whole study, the authors should be able to articulate their "improved understanding". In short, the authors should be able to frame the overall article better.

Response to R2C12: As shown in Fig 3 for the xylem samples, the simulated xylem samples were not as depleted for Ic-excess as the measured compositions (also see P18 Ln. 9-10). Simulations were unable to accurately simulate the fractionation in either the fast or slow domain, and the large fractionation was not observed in the

Printer-friendly version

Discussion paper 
soil waters. The authors recognize that there are limitations with the current model structure; however, we were demonstrating that there are numerous theories for why the fractionation of xylem water occurs. The occurrence of fractionation in xylem water is not site specific (eg. McCutcheon et al., 2017). The discussion sections (4.1 and 4.2) will be modified to clarify the (1) linkages between the non-physical nature of the model structure to physical mechanisms, (2) further use of SAS functions to test the theories of eco-hydrological separations, and (3) the wider implications of the SAS functions of root-uptake and evaporation (e.g. Fig. 6).

\section{References}

Kuppel, S., Chevallier, F., and Peylin, P.: Quantifying the model structural error in carbon cycle data assimilation systems, Geosci. Model Dev., 6, 45-55, doi: 10.5194/gmd6-45-2013, 2013.

McCutcheon, R., McNamara, J., Kohn, M., and Evans, S.: An evaluation of the ecohydrological separation hypothesis in a semiarid catchment, Hydro. Proc., 31(4), 783799, doi: 10.1002/hyp.11052

Interactive comment on Hydrol. Earth Syst. Sci. Discuss., https://doi.org/10.5194/hess-201857, 2018. 